the 9 patients with renal remission, 4 relapsed. Finally, 4 patients required dialysis, including 1 death.

Conclusion The presence of intrarenal B cells rarely forms TLO in lupus nephritis. Due to the small sample size, we were unable to determine their prognostic role. Nevertheless, we report here the longer time to renal remission for grade 3 and 4 infiltrates; prospective studies with repeated renal biopsies are needed to better characterize their relationship to disease progression.

Funding None

\section{O22 CORRELATION BETWEEN INTERSTITIAL CD8+ T CELL INFILTRATION AND FIBROTIC PROCESSES IN A MOUSE MODEL OF LUPUS NEPHRITIS}

1,2 Pauline Montigny, ${ }^{1}$ Aurélie Degroof, ${ }^{3}$ Davide Brusa, ${ }^{1,4}$ Frédéric Houssiau,
${ }^{1,4}$ Bernard Lauwerys. ${ }^{1}$ Pôle de Pathologies Rhumatismales Systémiques et Inflammatoires,
UCLouvain, Louvain; ${ }^{2}$ Service de Rhumatologie, CHU UCL Namur, Yvoir; ${ }^{3}$ Plateforme de
Cytométrie de Flux, Institut de Recherche Expérimentale et Clinique, UCLouvain, Louvain;
${ }^{4}$ Service de Rhumatologie, Cliniques Universitaires Saint Luc, Bruxelles, Belgium

10.1136/lupus-2020-eurolupus.33

Background Tubulo-interstitial damage during lupus nephritis $(\mathrm{LN})$ is associated with poor renal prognosis in the long run. Here, we describe the progression of tubulo-interstitial fibrosis and immune cells infiltration with emphasis on CD8 + T cells, in parallel with renal outcomes in a mouse lupus model.

Methods We collected blood, urine and kidneys from 39 B6/ Sle1.Sle2.Sle3 lupus-prone mice, before disease onset and at different stages of disease progression. RNA was extracted from kidneys and hybridized on Mouse Gene 2.0 ST exon arrays. Histopathological scores (NIH Activity and Chronicity Indexes) and digital quantification of fibrosis, IgGK deposits, $\mathrm{CD} 8+$ and $\mathrm{CD} 3+\mathrm{T}$ cells were performed on total kidney. Renal CD8 $+\mathrm{T}$ cell phenotypes were determined by flow cytometry. Plasma urea and albuminuria were measured by immunoenzymatic assays.

Results IgGк deposits, CD8 $+\mathrm{T}$ cell infiltration and interstitial fibrosis increase with the progression of renal disease, evaluated by histopathological scores and plasma urea. Further, digital quantifications allowed us to identify a significant correlation $(\mathrm{r}=0,45, p=0,011)$ between local CD8 $+\mathrm{T}$ cell population and fibrosis, while total $\mathrm{CD} 3+$ cells population and

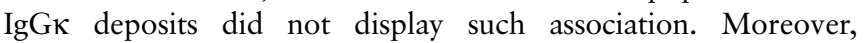
characterization of $\mathrm{CD} 8+\mathrm{T}$ cell subpopulations showed that fibrosis is more specifically linked to effector functions of
CD8 $+\mathrm{T}$ cells. Transcriptomic analyses supported this association, with a high correlation coefficient between mean expression of effector functions transcripts and the presence of a fibrotic signature $(r=0,92, p<0,0001)$.

Conclusions Our results support the association between CD8 $+\mathrm{T}$ cell tubulo-interstitial infiltration and renal outcomes in a mouse lupus model. Further, a strong correlation is identified between effector functions of CD8 $+\mathrm{T}$ cells and fibrotic processes, opening new avenues of research in the pathogenesis of LN.

Acknowledgements This work was supported by F.R.S-F.N.R.S and by Fondation Roi Baudouin

\section{O23 IDENTIFICATION OF PROTEIN-QUANTITATIVE TRAIT LOCI (PQTLS) IN THE INTERFERON SIGNALLING PATHWAY}

Christian Lundtoft, Pascal Pucholt, Johanna K Sandling, Lars Rönnblom, Niklas Hagberg. Dept. of Medical Sciences, Rheumatology, Uppsala University, Uppsala, Sweden

\subsection{6/lupus-2020-eurolupus.34}

Background Interferon (IFN)- $\alpha$ and IFN- $\gamma$ are important cytokines in the pathogenesis of systemic lupus erythematosus (SLE), and several of the genetic associations with SLE are found in genes that are fundamental for the IFN response (e. g. TYK2, STAT4, IRF5). This study aimed to define the genetic regulation of the IFN system, and to link disease-associated SNPs to alterations in the IFN system.

Methods Peripheral blood mononuclear cells from 303 healthy individuals were stimulated with IFN- $\alpha$ or IFN- $\gamma$ (figure 1). Basal levels of IFN-receptors (IFNAR2 and IFNGR1) and IFN-induced phosphorylation of STAT1 and STAT4, expression of CXCL9, CXCL10, HLA-ABC and HLA-DRPQ was determined in 6 cell subsets using flow cytometry. Each readout was mapped as a pQTL using 3.4 million SNPs with a minor allele frequency $\geq 5 \%$ (Illumina Global Screening Array with subsequent genome-wide imputation) in an additive model correcting for covariates. pQTLs were probed for overlap with GWAS SLE-associated SNPs.

Results We identified 8 genome-wide significant pQTLs $(\mathrm{p}<5.0 \mathrm{e}-8), 3$ of which were associated with basal IFN receptor levels, 3 with IFN- $\alpha$, and 2 with IFN- $\gamma$-induced traits. One pQTL affected protein expression in cis (IFNAR2), whereas the other were trans-pQTLs. The strongest association was observed for a SNP in HLA-A that affected IFNAR2 level

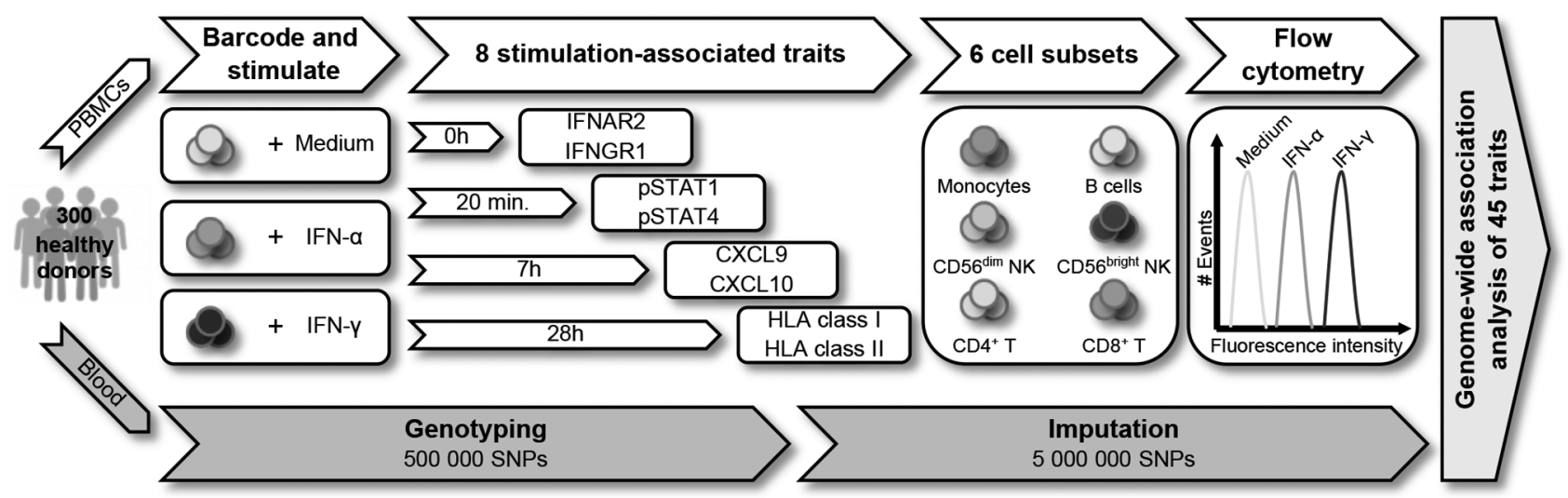

Abstract 023 Figure 1 Graphical study design 